\title{
Optical In-Building Network Techniques
}

\author{
A.M.J. Koonen, H. Yang, H.-D. Jung, S.C.J. Lee, E. Tangdiongga, C. Okonkwo, \\ H.P.A. van den Boom \\ COBRA Institute, Eindhoven University of Technology, \\ P.O. Box 513, NL5600MB Eindhoven, The Netherlands \\ e-mail: a.m.j.koonen@tue.nl
}

\begin{abstract}
By deploying optical fiber, versatile in-building networks which integrate wired and wireless services can be realized. Advanced signal modulation techniques enable high-capacity services over multimode fiber, and dynamic wavelength routing can improve the system's efficiency.
\end{abstract}

\section{INTRODUCTION}

In order to offer a set of different services, nowadays a mixture of networks each optimized for a specific subsets of services are being deployed in today's residential homes and in (semi-)professional buildings such as hospitals, office buildings, conference centers, etc. Twisted pair copper line networks are delivering voice telephony and facsimile services, coaxial cable networks deliver TV and radio broadcast services, CAT-5 cable networks provide data connections between $\mathrm{PC}$-s and other data processing/storage modules, wireless LANs are interconnecting laptop computers, PDAs and other wireless terminals, infrared control is used for remotely steering TV and audio equipment, and some other wired networks may be in place for domotica applications such as heating control, burglar and fire alarms, camera surveillance, etc. Obviously, such a jungle of networks complicates maintenance and the introduction of new services as well as the creation of links between services on different networks (e.g., running a movie from your PC on your TV network). Hence, we foresee considerable benefits in establishing a single in-building network which can act as a universal transport backbone network; see Fig. 1. Such a network can interface with the access network(s) through a single unit, the Residential Gateway (RG), which can host a lot of signal translation and storage functions. Because of its signal format transparency, immunity to electromagnetic interference, small size and huge bandwidth, optical fiber is very attractive as the transport medium in the backbone network. Single-mode fiber (SMF) obviously has the highest performance characteristics in terms of a very low loss per unit of length and an ultra-high bandwidth*length product; however, its delicate handling makes it costly to install. More recently, bend-insensitive SMF types have been introduced which relax some of the installation issues. On the other hand, in-building link lengths are relatively short implying that a top-notch fiber is not necessary. Multimode silica fiber (MMF) is much easier to install due to its much reduced sensitivity to connector and splice misalignments. In particular, large-core plastic optical fiber (POF) is very easy to cut and interconnect, thus even lending itself for do-ityourself (DIY) installation which is highly preferred for lowcost installation by (unskilled) home owners. On the other hand, high-NA step-index PMMA POF has a high amount of modal dispersion and thus quite a limited bandwidth. Advanced signal modulation techniques are needed in order to carry high data rates over such fiber.

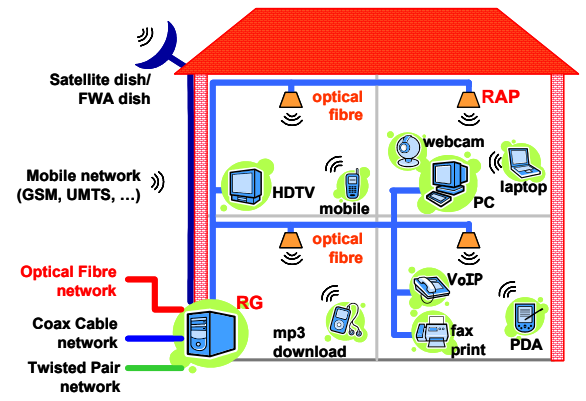

Fig. 1 Integrating wired and wireless services in a single optical fiber network

\section{IN-BUILDING FIBER NETWORK ARCHITECTURES}

Several basic architectures may be considered for the inbuilding network. In point-to-point (P2P), individual fibers run from the $\mathrm{RG}$ to every room/wall outlet. In point-tomultipoint (P2MP), one fiber link departing from the RG may be split into several branches each connecting to a wall outlet. Examples of P2MP topologies are a bus architecture (with a branching point per wall outlet) and a tree architecture (with a common branching point per floor, from where individual fibers run to the wall outlets). The splitting may be opaque (i.e. including $\mathrm{O} / \mathrm{E} / \mathrm{O}$ conversion) or all-optical. In multipointto-multipoint (MP2MP), interconnections may be established between wall outlets via a star coupler (near the RG) without intervention of the RG. A first analysis has been made of the network installation costs, taking into account the costs of the ducts, the cables, and the (opaque) network splitters [1] . As a conclusion, it was found that for small low-rise buildings (such as typical residential homes) a P2P architecture using DIY POF is the most attractive. For larger high-rise buildings (such as office buildings, hospitals, ...) a P2MP bus architecture may be the best choice. For easy upgrading, an all-optical P2MP network with optical power splitting or wavelength routing modules may be the best; due to the very limited availability of multimode passive network modules, such a network needs to use SMF. On the other hand, as this 
concerns mostly professional buildings, the higher costs of SMF network installation may not be a serious drawback.

\section{BROADBAND SERVICES OVER MULTIMODE FIBER}

Obviously, the largest technological challenge lies in providing high-capacity wired as well as wireless services over a highly dispersive MMF/POF network.

The signaling line rate for a given data rate can be significantly reduced by multi-carrier techniques in combination with multi-level/-phase modulation formats per carrier. We have shown that Discrete Multitone Modulation (DMT) with adaptive bit loading is a very powerful technique to handle highly dispersive fiber links. Using off-line signal processing, we demonstrated 4Gbit/s with a $667 \mathrm{~nm}$ VCSEL over 50 meters of $0.9 \mathrm{~mm}$ core graded-index POF, with 160 carriers loaded with QAM-16 down to OOK depending on the carrier's SNR [2] . A real-time 1.25Gbit/s DMT transmitter was built and operated successfully over 10 meters of $1 \mathrm{~mm}$ core step-index POF using a fast FPGA and an RC-LED [3] . For delivery of broadband wireless services, radio-over-fiber (RoF) supported wireless pico-cells are attractive. We have shown that our Optical Frequency Multiplying (OFM) technique is able to generate very pure microwave carriers and deliver these over dispersive MMF/POF links [4] . 120 $\mathrm{Mbit} / \mathrm{s}$ on a single subcarrier in QAM-64 format has been transported over $4.4 \mathrm{~km} 50 \mu \mathrm{m}$ core silica graded-index MMF, at a carrier frequency of $17.2 \mathrm{GHz}$. Multi-tone techniques as used in wireless standards can also be handled; a 52-carriers OFDM 16-QAM 36Mbit/s signal at $18.3 \mathrm{GHz}$ has been conveyed over 950 meters of silica $50 \mu \mathrm{m}$ core silica gradedindex MMF, including intermediate wavelength conversion by cross-gain modulation in a single SOA [5] ; see Fig. 2.

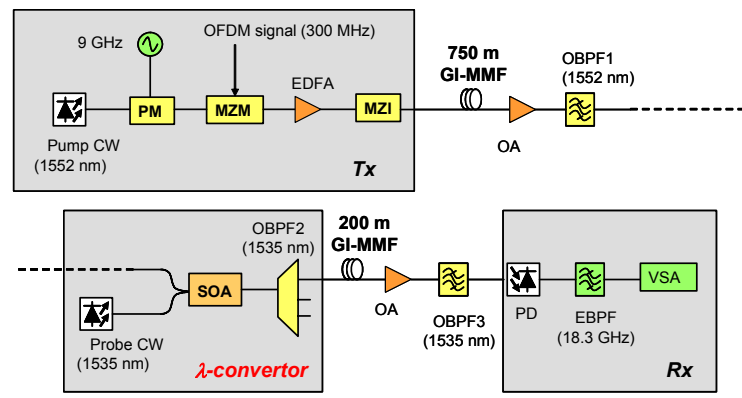

Fig. 2 Wavelength-conversion of OFM RoF signals

\section{DYNAMIC NETWORK RECONFIGURATION}

The traffic loads, both by wired and wireless services, may vary across the building during the day, in particular in larger buildings. E.g., in university buildings students will close laptop communication sessions after a lecture at one place, move to another place, and open a new session. In airport departure halls, traffic loads may be high at a gate when many people waiting for their flight are making phone calls and using internet access, while somewhat later this load may occur at an other gate. In order to deploy the installed resources the most efficiently, unused network capacity may be directed to those places where an actual high demand exists. Optical wavelength routing in an all-optical network architecture is a powerful way to establish this for various types of services. By routing of multiple wavelength channels in the network, different network connection patterns for different types of services can be set. A subnetwork for highcapacity wired services using multilevel multitone modulation formats can be set up at one wavelength, while another subnetwork for radio-over-fiber picocells can be configured at an other wavelength. Fig. 3 illustrates how mobile wireless devices (MD-s) may interconnect via radioover-fiber links which are routed by means of wavelength conversion in the RG and wavelength add-drop nodes $(\lambda$-ADN-s) feeding the radio access points (RAP-s) in each room. Recently, we showed all-optical multicast routing of RoF signals by cross-gain wavelength conversion in a single SOA followed by an arrayed waveguide grating router [6] .

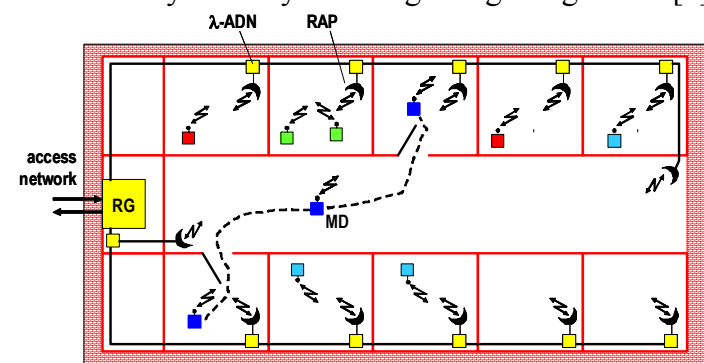

Fig. 3 Reconfigurable radio-over-fiber network

\section{CONCLUSIONS}

Optical fiber techniques are well suited for integrating wired and wireless services in a single in-building network. Multitone multi-level/-phase modulation formats enable high data rate wired services over multimode fiber, whereas optical frequency multiplying enables high-capacity picocell wireless services over the same fiber. Capacity-on-demand can be provided by dynamically controlled wavelength routing. Funding from the European Commission in the FP7 projects ALPHA, POF-PLUS and BONE is gratefully acknowledged.

\section{REFERENCES}

[1] T. Koonen, A. Pizzinat, E. Tangdiongga, P. Guignard, H.-D. Jung, H. van den Boom, "In-building optical network architectures for converged services delivery", Proc. NOC 2009, Valladolid, June 10-12, 2009

[2] H. Yang, E. Tangdiongga, S. C. J. Lee, S. Randel, H.P.A. van den Boom, A.M.J. Koonen, "4 Gbit/s over 50-m large core diameter GI-POF using low-cost VCSEL", accepted for ECOC2009

[3] S.C.J. Lee, F. Breyer, D. Cardenas, S. Randel, T. Koonen, "Real-time implementation of a 1.25-Gbit/s DMT transmitter for robust and low-cost LED-based plastic optical fiber applications", accepted for ECOC 2009

[4] A.M.J. Koonen, M. García Larrodé, "Radio-over-MMF techniques - Part II: microwave to millimeter-wave systems", IEEE J. of Lightwave Technol., 2008, Vol. 26, No. 15, pp. 2396-2408

[5] H. Yang, H.-D. Jung, Y. Zheng, B. Huiszoon, J.H.C. van Zantvoort, E Tangdiongga, A.M.J. Koonen, "OFDM Radio-over-Fibre Systems Employing Routing in Multi-Mode Fibre In-Building Networks", Proc. ECOC 2008, Brussels, Sep. 21-25, 2008, paper Tu.4.F.6

[6] H.-D. Jung, C. Okonkwo, E. Tangdiongga, T. Koonen, "All-optical multicasting of millimetre-wave signals using optical frequency multiplication technique for in-building networks", accepted for ECOC 2009 